\title{
Listening carefully: increased perceptual acuity for species discrimination in multispecies signalling assemblages
}

\author{
Anna Bastian*, David S. Jacobs \\ Department of Biological Sciences, University of Cape Town, Cape Town, South Africa
}

\section{A R T I C L E I N F O}

\section{Article history:}

Received 1 August 2014

Initial acceptance 23 September 2014

Final acceptance 11 November 2014

Published online

MS. number: 14-00625R

\section{Keywords:}

acoustic assemblages

acoustic communication

acoustic communication hypothesis

bats

echolocation

functional extension

habituation-dishabituation

receivers' perception acuity

Rhinolophus

species discrimination
Communication is a fundamental component of evolutionary change because of its role in mate choice and sexual selection. Acoustic signals are a vital element of animal communication and sympatric species may use private frequency bands to facilitate intraspecific communication and identification of conspecifics (acoustic communication hypothesis, ACH). If so, animals should show increasing rates of misclassification with increasing overlap in frequency between their own calls and those used by sympatric heterospecifics. We tested this on the echolocation of the horseshoe bat, Rhinolophus capensis, using a classical habituation-dishabituation experiment in which we exposed $R$. capensis from two phonetic populations to echolocation calls of sympatric and allopatric horseshoe bat species (Rhinolophus clivosus and Rhinolophus damarensis) and different phonetic populations of $R$. capensis. As predicted by the ACH, R. capensis from both test populations were able to discriminate between their own calls and calls of the respective sympatric horseshoe bat species. However, only bats from one test population were able to discriminate between calls of allopatric heterospecifics and their own population when both were using the same frequency. The local acoustic signalling assemblages (ensemble of signals from sympatric conspecifics and heterospecifics) of the two populations differed in complexity as a result of contact with other phonetic populations and sympatric heterospecifics. We therefore propose that a hierarchy of discrimination ability has evolved within the same species. Frequency alone may be sufficient to assess species membership in relatively simple acoustic assemblages but the ability to use additional acoustic cues may have evolved in more complex acoustic assemblages to circumvent misidentifications as a result of the use of overlapping signals. When the acoustic signal design is under strong constraints as a result of dual functions and the available acoustic space is limited because of cooccurring species, species discrimination is mediated through improved sensory acuity in the receiver. (C) 2015 The Association for the Study of Animal Behaviour. Published by Elsevier Ltd.
Communication plays a crucial role in almost all aspects of an animal's life (e.g. Maynard Smith \& Harper, 2003) and is especially important for species discrimination (Bradbury \& Vehrencamp, 2011; Ryan \& Rand, 1993). It transmits information within a species as well as across species and may have evolved as a product of species coexistence (Li et al., 2013). Discriminating species is important in interactions with heterospecifics allowing identification of competitors, predators and prey, whereas the recognition of conspecifics is a prerequisite for any species-specific interactions, especially for mate choice (Jones, 1997; Sandoval, Méndez, \& Mennill, 2013; Slabbekoorn \& Smith, 2002; Wilkins, Seddon, \& Safran, 2013). Understanding how communication signals

\footnotetext{
* Correspondence: A. Bastian, UCT, Upper Campus, Private Bag X3, Rondebosch, 7701, Cape Town, South Africa.

E-mail address: anna.bastian@mail.de (A. Bastian).
}

originate and are modified over evolutionary time is therefore crucial to our understanding of the processes that generate biodiversity (Mendelson \& Shaw, 2012). It is likely that communication systems evolved from systems used for other purposes (Monteiro \& Podlaha, 2009; Tinbergen, 1952), such as the function of feathers first used for insulation being extended so that they also function as visual signals, for example in courtship displays (Cowen, 2005). Particularly, knowledge of processes involved in the evolution of dual functions for a single trait can provide insight into how phenotypic diversity in both form and function is generated from existing variation.

Echolocation may provide us with an opportunity to investigate such functional extension of a trait. Echolocation is primarily used for orientation and food acquisition in echolocating bats, birds and whales (Brinkløv, Fenton, \& Ratcliffe, 2013; Schnitzler, Moss, \& Denzinger, 2003; Thomas, Moss, \& Vater, 2004) but there is increasing evidence that it also functions as a means of 
communication (Gregg, Dudzinski, \& Smith, 2007; Jones \& Siemers, 2010). In the context of species discrimination, communication cues have to be unambiguous and represent a reliable badge for the species. Such species-specific cues are present in the vocalizations of many animal groups including insects, anurans, birds and mammals (primates: Seyfarth, Cheney, \& Marler, 1980; anurans: Duellman \& Pyles, 1983; birds: Catchpole \& Slater, 2008; insects: Pennetier, Warren, Dabiré, Russell, \& Gibson, 2010). Vocalizations are often a crucial signal in mate choice (Anderson, Ambrose, Bearder, Dixon, \& Pullen, 2000; Braune, Schmidt, \& Zimmermann, 2008; Charlton, Huang, \& Swaisgood, 2009; Vannoni \& Mcelligott, 2007) as they can provide information about the sender which is used by the receiver to evaluate the mate's intention, compatibility and quality. The voice of mammals, for example, is often an honest cue which allows an individual to assess the body size or mass of the sender (Fitch, 2006; Liebermann \& Blumstein, 1991). Among echolocating mammals bats are ideal candidates for studies on echolocation in the context of communication because most species form groups with complex social structures (Kulzer, 2005) in which many interactions are managed by acoustic signals (Altringham \& Fenton, 2003; Fenton, 1985). The acoustic structure of their echolocation calls has a complex frequency-time contour and there are many different types of calls (Maltby, Jones, \& Jones, 2010) providing sufficient variation to encode multiple cues. Furthermore, echolocation calls contain diagnostic information about the sender which can be useful for others and, as a frequently available signal, echolocation transmits information free of additional costs to a receiver (Dechmann, Wikelski, Noordwijk, Voigt, \& Voigt-Heucke, 2013). In echolocating bats, the relationship between echolocation call frequency and body size is well established (Jacobs, Barclay, \& Walker, 2007; Jones, 1999), and echolocation calls often carry species-specific signatures, individual signatures, population-specific signatures and sex-specific signatures (Jones \& Siemers, 2010).

Several recent playback studies have provided evidence that conspecific bats are able to extract information encoded in the echolocation calls of other bats such as species membership, familiarity and sex (Dorado Correa, Goerlitz, \& Siemers, 2013; Knörnschild, Jung, Nagy, Metz, \& Kalko, 2012; Schuchmann, Puechmaille, \& Siemers, 2012; Voigt-Heucke, Taborsky, \& Dechmann, 2010) and have recently revealed a role in mate choice (Puechmaille et al., 2014). However, because echolocation has evolved primarily for orientation and food acquisition (Schnitzler et al., 2003) species assemblages that are composed of ecologically similar bat species, and which therefore have similar echolocation call structures (Denzinger \& Schnitzler, 2013), should partition the acoustic characteristics of their echolocation calls so that the calls retain their species specificity (Duellman \& Pyles, 1983; Heller \& von Helversen, 1989). The concept of acoustic divergence of signals for species discrimination in multispecies assemblages to avoid misidentification as a result of the use of confusingly similar calls (Amézquita, Flechas, Lima, Gasser, \& Hödl, 2011; Tobias, Planqué, Cram, \& Seddon, 2014) is well established in animal communication (Grant \& Grant, 2010; West-Eberhard, 1983). This idea was first advanced by Duellman and Pyles (1983) for anurans and Heller and von Helversen (1989) for bats and later named the acoustic communication hypothesis $(\mathrm{ACH}$, Jacobs et al., 2007) which is similar to the spectral partitioning hypothesis coined by Amézquita et al. (2011). Both the ACH and the spectral partitioning hypothesis propose that sympatric animal species each uses a 'private frequency channel' to facilitate intraspecific communication and identification of conspecifics (Heller \& von Helversen, 1989). This is supported by the divergence in the echolocation frequency of some bat species possibly as a consequence of the presence of other species (Heller \& von Helversen,
1989; Jacobs et al., 2007; Kingston, Jones, Zubaid, \& Kunz, 2000; Kingston \& Rossiter, 2004; Russo et al., 2007). This acoustic divergence among signallers has also been found in morphologically cryptic species living in sympatry (Guillén, Juste, \& Ibañez, 2000; Jones \& Siemers, 2010; Jones \& Van Parijs, 1993; Kingston et al. 2001; Thabah et al., 2006). However, a test of the ACH would also have to incorporate an investigation of the perception and discrimination ability of the receiver.

In this study we used the horseshoe bat, Rhinolophus capensis, to investigate the role of echolocation in communication in the context of the ACH. We chose a classical habituation-dishabituation experiment (Eimas, Siqueland, Jusczyk, \& Vigorito, 1971) in which we exposed $R$. capensis to recorded calls of two sympatric horseshoe bat species (Rhinolophus clivosus and Rhinolophus damarensis) and different phonetic populations of $R$. capensis. In these assemblages we have populations of the same species using different echolocation frequencies as well as different heterospecifics using overlapping frequencies. This natural system provides an excellent opportunity to test whether $R$. capensis discriminates between different species and populations on the basis of their echolocation calls. If acoustic divergence in the echolocation frequencies of $R$. capensis is a result of selection favouring the use of private frequency bands as proposed by the $\mathrm{ACH}, R$. capensis should show increasing rates of misclassification with increasing overlap between its own calls and those used by sympatric heterospecifics. This concomitantly means that individuals of $R$. capensis from the different phonetic populations should have difficulty recognizing each other as belonging to the same species if they use calls of dissimilar frequency. In addition this system allows us to test whether peak frequency is the only parameter used by bats to discriminate between species.

\section{METHODS}

\section{Study Animal}

Rhinolophus capensis (Cape horseshoe bat) has a wide distribution along the coastal belt of South Africa's Cape (Monadjem, Taylor, Cotterill, \& Schoeman, 2010). This species emits resting frequency echolocation calls (RF, calls emitted by rhinolophid bats when stationary and hunting from a perch; Neuweiler et al., 1987; Schnitzler, 1968) that vary by more than $10 \mathrm{kHz}$ across its distribution range (Fig. 1; Odendaal, Jacobs, \& Bishop, 2014). The lowest resting peak frequency, $75 \mathrm{kHz}$, is found in the northwestern part of its distribution, and the highest, $86 \mathrm{kHz}$, in the southeast. These phonetic populations co-occur with various other horseshoe bat species, namely R. damarensis (Jacobs et al., 2013) in the northwest and $R$. clivosus in the southern and eastern part (Jacobs et al., 2007). When both juveniles and adults are considered, $R$. clivosus calls at 87-92 kHz (Jacobs, n.d.) and $R$. damarensis at $79-87 \mathrm{kHz}$ (Jacobs et al., 2013). In both cases $R$. capensis populations echolocate on average $2-9 \mathrm{kHz}$ lower than the respective sympatric heterospecific. This natural system provides an excellent opportunity to test the $\mathrm{ACH}$.

\section{Study Sites}

The experiments were done at two sites in South Africa: De Hoop Nature Reserve on the southern coast of South Africa (March 2012 and October 2012) which represents a geographically central population with RFs at $85 \mathrm{kHz}$ and a second more remote population at the Orange River near Lekkersing in the extreme northwestern corner of South Africa on the border with Namibia (November 2012). The latter population uses considerably lower RFs of $75 \mathrm{kHz}$. 


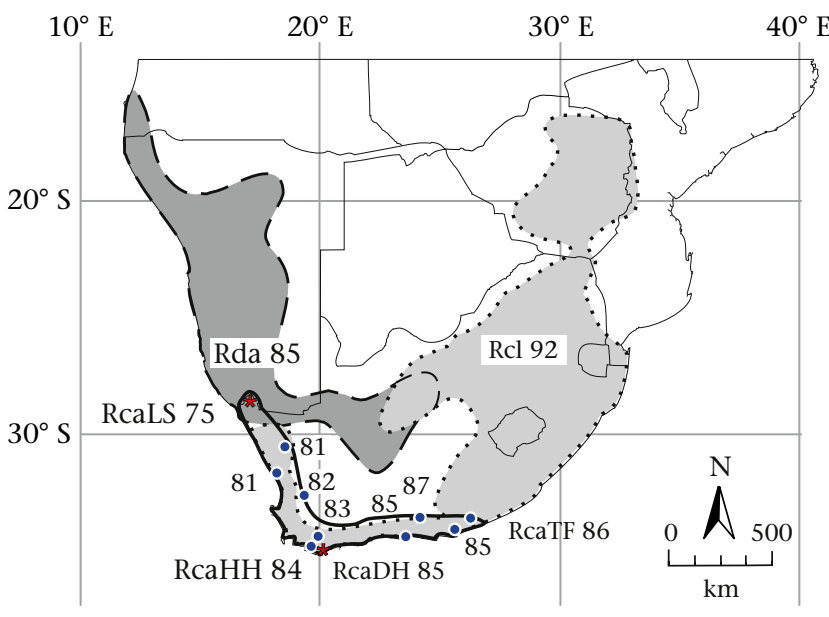

Figure 1. Map of southern Africa indicating the distributions of three horseshoe bat species, Rhinolophus damarensis in the west (Rda, dashed line), $R$. clivosus in the east (Rcl, dotted line) and R. capensis in the south (Rca, solid line). Circles indicate sample sites for $R$. capensis, whereas the two asterisks refer to the two study sites. Numbers indicate peak resting frequencies. $\mathrm{LS}=$ Lekkersing, $\mathrm{HH}=$ Heidehof, $\mathrm{DH}=\mathrm{De}$ Hoop, $\mathrm{TF}=$ Table Farm. The distribution of $R$. capensis is adapted from Odendaal et al. (2014) including the additional resting peak frequencies across the range. The distribution of $R$. damarensis is adapted from Jacobs et al. (2013) and the distribution from $R$. clivosus from Monadjem et al. (2010).

\section{Ethical Note}

The treatment of animals in this study met the requirements of ethical guidelines of the Federation for Laboratory Animal Science Associations (FELASA), the American Society of Mammalogists and the South African National Standard (SANS, 2008; Guillen, 2012; Sikes \& Gannon, 2011). All experiments were done with permission of local authorities (2399/2102; 0035-AAA007-00081) and the Animal Ethics Committee of the University of Cape Town (2012/ V33/DJ) and conducted by a trained person (A.B., FELASA-B certificate).

\section{Capture, Release, Housing and Animal Care}

Bats were caught with mist nets (Ecotone monofilament, $20 \times 20 \mathrm{~mm}$ mesh size, Avinet, Dryden. NY, U.S.A.) at the entrance to two caves (Guano Cave in De Hoop Nature Reserve and Wondergat Cave at the Orange River) as the bats emerged to forage. Only adult males and nonlactating adult females were kept and immediately brought to housing facilities in soft cotton bags (a maximum 30 min drive from each of the caves). After experiments, bats were released back into their respective caves at night. We observed the bats after releasing them to make sure they showed no signs of weakness and could fly off easily.

We kept the bats in small mixed-sex groups in a transport cage lined with soft wire mesh ( $40 \times 25 \mathrm{~cm}$ and $25 \mathrm{~cm}$ high) with up to six bats for up to 3 nights or in a two-person tent $(105 \times 105 \mathrm{~cm}$ and $205 \mathrm{~cm}$ high) with up to 12 bats for 6 nights. The tent was equipped with a water bowl and towels to provide perches and hiding places for the bats. The cage/tent was housed in the respective research houses inside a quiet room which we darkened and kept at ambient temperature and humidity for most of the time but occasionally heated (in March) and cooled (in November). Handling of bats was kept to a minimum; we offered them water twice per day and only handled them again prior to experiments. We used headlights (at lowest illumination) when handling bats; otherwise it was completely dark. We noted the weight of each bat each night to make sure bats were fed sufficiently. Immediately before experiments we gave them water and up to 10 mealworms (Tenebrio molitor larvae) to increase motivation. Immediately after the experiments we fed them with 30-50 mealworms and again gave them water. To ensure high nutrition of the diet the mealworms were fed with fresh vegetables and fruit, dry dog food and mineral powder for 4 weeks prior to field trips.

\section{Experimental Approach}

We did a habituation-dishabituation playback experiment to test whether $R$. capensis individuals from two different localities in South Africa, Lekkersing (LS) in the northwest and De Hoop (DH) in the south, perceive differences in the acoustic structure of echolocation calls from different phonetic populations of their own species and other sympatric and allopatric horseshoe bat species (i.e. $R$. damarensis and R. clivosus). This experiment is well suited to test whether two or more stimuli classes are perceived by the study subjects as being different and thus discriminated (Bouton, 2007; Simmons, Popper, Fay, \& Gerhardt, 2003). One stimulus class was played back continuously until the study subject no longer reacted to it, i.e. it was habituated to this stimulus. Habituation is defined as a gradual decline in response following repeated exposure (Bukatko \& Daehler, 2003; Fantz, 1964). This is needed to have a common zero baseline of activity across individuals against which to evaluate the discrimination response. Once the bat was habituated a test stimulus was presented. If the bat became active again after switching to the test stimulus, it indicated a dishabituation (Bukatko \& Daehler, 2003). Dishabituation is defined as a rapid recovery from habituation (Rankin et al., 2009) based on the ability to discriminate between the two stimuli classes.

Our set of stimuli consisted of six acoustic stimuli classes each representing one of the four phonetic populations of $R$. capensis as well as one of the other horseshoe bat species, $R$. clivosus and $R$. damarensis, each co-occurring with one of our $R$. capensis test populations. More precisely, our six playbacks contained calls from (1) R. capensis from De Hoop which echolocates at $85 \mathrm{kHz}$ (abbreviated as RcaDH85) in the south, (2) R. capensis from Heidehof (RcaHH84) also in the south, (3) R. capensis from Table Farm (RcaTF86) in the southeast, and (4) $R$. capensis from Lekkersing (RcaLS75) in the northwest, as well as from (5) R. clivosus which echolocates at $92 \mathrm{kHz}$ (Rcl92) and co-occurs with RcaDH85 at De Hoop, and (6) R. damarensis echolocating at $85 \mathrm{kHz}$ (Rda85) and cooccurring with RcaLS75 at Lekkersing). Figure 1 shows a map of South Africa indicating the distribution ranges of the three species, the geographical localities where the respective stimuli classes were recorded and the two study sites.

\section{Stimulus Generation}

The echolocation calls used in this study were previously recorded in a standardized way (Odendaal et al., 2014). For all playback files we chose good quality echolocation calls with a high signal-to-noise ratio. Because horseshoe bats tune into their resting peak frequency after a period of silence (Schuller \& Suga, 1976; Siemers, Beedholm, Dietz, Dietz, \& Ivanova, 2005) we chose calls emitted after the first 10 calls in each recording. We decided to use only calls from males because the RF differs between the sexes in $R$. capensis (Odendaal \& Jacobs, 2011). To minimize effects of pseudoreplication, which is the possibility that bats may memorize a set of individuals rather than forming a template of the characteristic call of the population (problem of pseudoreplication; Mc Gregor et al., 1992), we used the highest possible number of individuals representing each phonetic population and species. We measured six spectral and three temporal parameters of the echolocation calls (see Audio analysis below and the Results). Parameter measurements of the six playback classes are given in 
Table A1 in the Appendix. A discriminant function analysis (DFA) showed that the calls used in the two playbacks from the two test populations, RcaDH85 and RcaLS75, are indeed representative of the respective phonetic population (Fig. 2). The DFA was performed on factor scores of a principal component analysis to obtain a set of uncorrelated acoustic variables. The first four principal components explained $84 \%$ of the variance and represented mainly the frequency, entropy and duration parameters (PC1: peak frequency of the dominant second harmonic, i.e. $R F(-0.94)$; peak frequency of the third harmonic $(-0.87)$; peak frequency of the first harmonic $(-0.79)$; PC2: minimum frequency of final FM component $(0.84)$ and entropy of the call $(-0.75)$; PC3: duration of call $(-0.79)$ and distance from start of the call to its maximum energy (disttomax; -0.78); PC4: intercall interval (0.88)). The stepwise canonical DFA on the four PCs (Wilks' $\lambda=0.00945$, approximately $F_{20,296}=455.4$, $P \leq 0.001$ ) indicated that function 1 contributed most to the discrimination between the six playback classes (partial Wilks' $\lambda=0.04)$, followed by function $2(0.025)$, function $3(0.56)$ and function 4 (0.77). Accordingly, function 1 is weighted most heavily by PC1 (coefficient 1.37; RF) and PC3 (coefficient - 0.79; call duration and disttomax) and explains $88 \%$ of the variance. Function 2 is marked by PC2 (1.03; minimum frequency of final FM component, i.e. minimum frequency at the end of the call and call entropy) and together both functions explain $99.5 \%$. This increases to $99.9 \%$ if function 3 is added ( 0.93 marked by PC4 reflecting the parameter intercall interval). Thus, the most discriminatory power was associated with the combined effects of peak frequency, call duration, disttomax, minimum frequency of final FM component and call entropy. The overall classification success was $84.5 \%$ for the six playback categories (LS: 100\%; DH 62\%; HH: 85\%; TF: 76\%; Rcl: 100\%; Rda: 65\%).

The RFs of our playbacks differed slightly from the published values as the available calls used to generate the playbacks shifted the mean RF between \pm 0.1 and $\pm 0.6 \mathrm{kHz}$. This deviation is well within the range of natural variability within the populations or species (Jacobs et al., 2013; Odendaal et al., 2014). In addition we

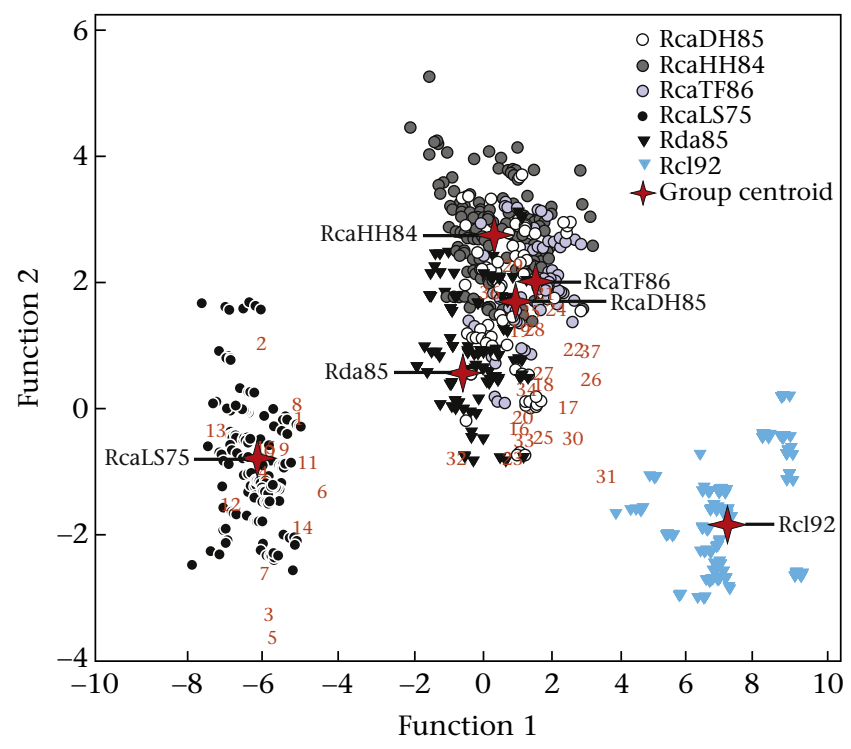

Figure 2. Plot of the first two functions of a discriminant function analysis (DFA) based on acoustic parameters of the playback stimuli and the experimental individuals. Each call of each playback class is plotted; numbers indicate the group centroid per individual. Each playback class is colour coded and each species is indicated with different symbols (see key). Individuals grouping with RcaLS75 playback calls on the left-hand side ( -4 to -8 on the $x$ axis) are test subjects from the study site Lekkersing, RcaLS75, whereas all other individuals on the right-hand side ( -1 to +4 on the $x$ axis) were from De Hoop, e.g. RcaDH85. calculated the coefficient of variation (as $\mathrm{CV}=(\mathrm{SD} /$ mean $) \times 100)$ to ensure that the selected calls and individuals did not come from the outer limits of the variability (CV would increase) which would increase the possibility of individual signatures in the playback and thereby the problem of pseudoreplication (Mc Gregor et al., 1992). All CVs were lower than the CVs of the published larger samples (RcaLS75: $1.3 \%$ in literature versus $0.8 \%$ this study; RcaDH85: $0.8 \%$ versus $0.5 \%$; RcaHH84: $0.6 \%$ versus $0.5 \%$; RcaTF86: $0.8 \%$ versus $0.6 \%$; Rcl: $0.9 \%$ versus $0.4 \%$; Rda: $1.6 \%$ versus $0.9 \%$ ).

In an attempt to keep the number of identical call replicates low we used 5-15 calls for each individual and applied a two-order randomization procedure by randomizing first the order of individuals and then the order of their calls. To achieve the required duration of playbacks with the limited number of available good quality calls per individual we repeated the unique calls randomly until we had $20 \mathrm{~s}$ long files per test stimulus class and $18.5 \mathrm{~min}$ long habituation files. We inserted each call file with its respective intervals into the playback. The habituation playback files consisted of 86 unique calls of nine individuals of $R$. capensis recorded at De Hoop and 58 unique calls of six individuals of $R$. capensis from Lekkersing. We chose four calls (RcaTF86, RcaDH85), five calls (RcaHH84, RcaLS75, Rda85) and six calls (Rcl92) per individual depending on the available number of individuals. For the test playback files, RcaDH85 and RcaLS75, we chose a set of new calls not used for the habituation playback file but from the same individuals used to compile the habituation playback file. The test files began with a $23 \mathrm{~ms}$ long series of habituation calls (a randomly chosen subset of the habituation calls), followed by the actual test calls with an averaged interval to the last habituation call. We decided to precede the test calls with habituation calls to ensure that any dishabituation reaction would be caused by the acoustic properties of the test stimuli and not by the onset of the test playback file because there was a short pause in playback while changing files. The series of test calls was followed by a control stimulus which was a $95 \mathrm{~ms}$ white noise and a low-frequency beep for synchronization of video and audio recordings $(0.7 \mathrm{kHz}$, $500 \mathrm{~ms}$ ) at the same intensity as the calls (Fig. 3).

The calls were semisynthesized, meaning that a natural call was used as a template to create a synthesized copy of it (Avisoft-SASLab Pro, v5.2, Avisoft Bioacoustics, Glienicke, Germany) to exclude any noise or recoding artefacts. All synthesized calls were normalized to the same intensity level. To estimate appropriate playback intensity we recorded two bats inside the box on the perch with a microphone placed next to the speaker through which the playbacks would be played with a medium sensitivity of the microphone (frequency gain on the detector one-third opened). We used the intensity of these recordings to adjust the intensity of the playback output by decreasing the volume of it inside the file until it was one-third louder than the recorded calls. This increased intensity was necessary to exclude the effect of a sensory bias on the discriminatory ability of the listeners. The auditory system of horseshoe bats contains an individualized 'acoustic fovea' (Neuweiler, Bruns, \& Schuller, 1980; Schuller \& Pollak, 1979) which is a narrow filter for increased frequency resolution that spans the individual's RF and covers approximately $8 \mathrm{kHz}$ (Neuweiler, 1990). Frequencies outside the foveal region have threshold levels comparable to other mammals which do not have an acoustic fovea (Neuweiler, 1990). Increasing the intensity of our playbacks minimized the possibility that differences in threshold levels could have resulted in a lack of response by the bats for those playback classes containing calls outside their foveal area. We tested the spontaneous response of three bats to the onset of a habituation playback to ensure that the bats' reaction towards the increased intensity of the playbacks was not a startle response (fast body contraction, cringe). All files began with a linear fade-in from $0 \mathrm{~dB}$ to maximum 
Habituation

Dishabituation

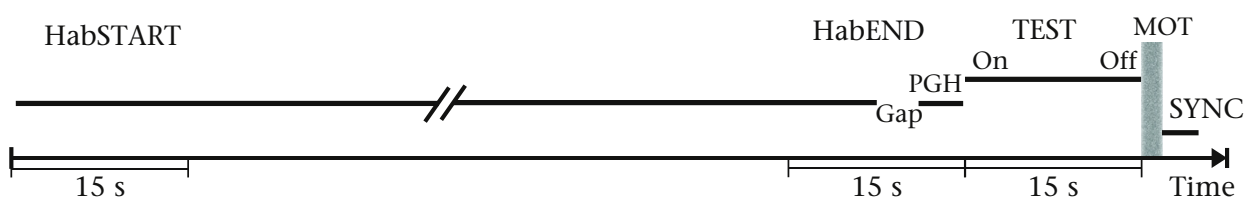

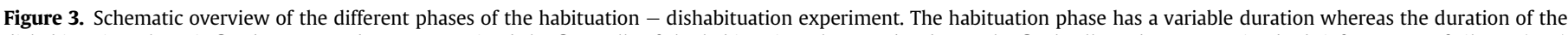

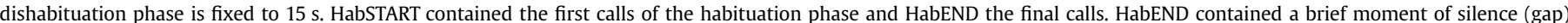

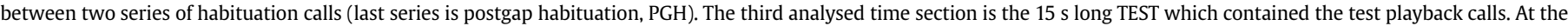
end of the TEST calls a motivational stimulus (MOT) was played back (white noise) followed by a synchronization beep (SYNC).

amplitude within the first call to avoid the clicking noise emitted by the loudspeaker when playback files began with maximum amplitude. The fading-in and -out was also used for the control and synchronization sounds (one-quarter of each element).

\section{Experimental Set-up}

Experiments took place in a custom-made experimental box measuring $77.5 \times 38.0 \mathrm{~cm}$ and $38.0 \mathrm{~cm}$ high (Genesisacoustics, Port Elizabeth, South Africa) similar to that used by Schuchmann and Siemers (2010) which was set up in a separate room. It was lined with $10 \mathrm{~cm}$ thick, high-frequency, sound-absorbing foam covered with glass cloth. It contained a perch for the bat on one end and a loudspeaker (ScanSpeak, Avisoft Bioacoustics) at the opposite end. A camcorder (DCR-SR42E Sony Corporation, Minato, Tokyo, Japan) with activated night-shot function was placed in one of the corners below the loudspeaker and pointing at the perch. An ultrasound detector (D1000X, Pettersson Elektronik, Uppsala, Sweden) with a calibrated microphone was placed in the other corner below the loudspeaker and pointed towards the perch. The loudspeaker was connected to an ultrasonic power amplifier (Avisoft Bioacoustics) and via a high-speed sound card (DAQCard-6062E, National Instruments, Austin, TX, U.S.A.) to a laptop (Hewlett and Packard Mobile Workstation, Palo Alto, CA, U.S.A. with the Microsoft Windows XP Professional, SP3, Microsoft Corporation, Redmond, CA, U.S.A. operating system). The camcorder was connected to a monitor outside the box to allow observation of the behaviour of the bat inside during experiments. To control the correct broadcasting of the playbacks we used Avisoft recorder software (rec-ni v4.2.15, Avisoft Bioacoustics). All settings on the equipment that could influence the output characteristics of the playbacks or the characteristics of the audio recordings were kept constant (amplifier volume, frequency gain of the bat detector) as well as the equipment components themselves.

\section{Experimental Procedure}

We used the following test regime: each bat listened to all stimuli classes after being habituated to calls from its own population on consecutive nights. The order of testing the bats was randomized and we ensured that each bat was not tested at the same time each night. The order of test stimuli was also randomized using the Box-Behnken Design, available in Statistica (v6.1, StatSoft Inc., Tulsa, OK, U.S.A.). The maximum duration for a trial was set to 40 min which was occasionally reached by individual bats on the first 2 nights.

The experiments began at around sunset. Each bat was brought to the experimental box in a small cotton bag and placed on the perch. If the bat crawled or flew off the perch we placed it back onto the perch until it stayed there. The playback started when the bat hung calmly at the perch and showed no visible movements for at least $20 \mathrm{~s}$. The habituation stimulus was played back and the immediate response of the bat was noted (reacted, did not react).
When the bat habituated to the habituation stimulus, i.e. showed no visible movements for $20 \mathrm{~s}$, we played back the test stimulus. Again, the response to the test and motivational control stimulus was noted (reacted, did not react). After each trial we left the bat for at least $30 \mathrm{~s}$ in the box before it was brought back to a separate room, where it was fed and later released into the housing quarters.

If any execution errors occurred during playbacks, the respective trial was repeated as the last trial for that bat. Trials were also repeated if a bat did not show a response to the habituation onset to prevent subsequent false negative responses to the test stimuli. In this case we stopped the habituation playback and started it again after $30 \mathrm{~s}$. If a bat did not react to the dishabituation stimulus and the motivational control played back at the end of each dishabituation stimulus we repeated the trial (again to control for false negatives). At both study sites we ran one to two trails per bat per night to shorten the period of captivity for each. Seven of a total of 32 bats listened to three trials in 1 night because of technical errors during the previous night. We ensured that playback classes were used in a balanced design across nights and individual bats that did multiple trials were used at different times of the night. At the study site in the northwest, LS, we limited the playback set to five trials because of the extremely high temperatures to reduce the risks of bats succumbing to heat exhaustion. Here we excluded the playbacks RcaTF86 and RcaHH84 and added the trial using RcaDH85calls (allopatric conspecific) for habituation and Rda85 calls (sympatric heterospecific) as the test stimulus to test whether bats can discriminate a different phonetic population of the same species from a different sympatric species using a very similar resting peak frequency. To facilitate direct comparison, the experiments were performed by the same person at each study locality.

\section{Recording of Experiments}

Each trial was synchronously recorded on video and audio from the moment each bat was placed on the perch inside the box until we took it out of the box at the end of the trial. Audio and video recordings were synchronized by using the low-frequency beep played back at the end of each test playback, SYNC in Fig. 3, which was recorded on both the audio line of the camcorder and the audio file of the bat detector to serve as the reference point between video and audio.

Audio recordings were done with a $385 \mathrm{kHz}$ sample rate, 16 bits depth and stored on internal CF cards of the bat detector as Waveform Audio File files (.wav). Large audio files were later split (Wave Splitter, v2.10, ClaudioSoft software, Ile-de-France, France) to allow analyses in the sound analysis software (Avisoft-SASLab Pro, v5.2, Avisoft Bioacoustics; BatSound Pro v3.31, Pettersson Elektronik $\mathrm{AB})$.

Video recordings were done using the internal infrared light of the camcorder (DCR-SR42E Sony Corporation) with the night shot function activated providing sufficient illumination for high-quality videos stored on the internal hard drive of the camcorder. Videos were transferred from the camcorder onto the laptop using the 
camcorder's software (Picture Motion Browser v2.0.06 Sony Corporation). Videos had to be converted (v3.0.057, Mangold Video Converter Pro, Mangold International, Arnstorf, Germany) to the Audio Video Interleave (.avi) format (video: AVC format, 8 bits depth, 25 fps frame rate, $265 \mathrm{Kbps}$ bit rate; audio: PCM format, 16 bits depth, $48 \mathrm{kHz}$ sampling rate, $1536 \mathrm{Kbps}$ bit rate) to ensure correct playback in the behavioural analysis software (InterAct v8.0, Mangold International).

\section{Analyses of Experiments}

\section{General statistics methods}

The global level of significance was $5 \%$ but we calculated the Bonferroni-corrected $P$ values to account for multiple comparisons. Full details of statistical analyses are given with each test, where necessary, in the Methods below and in the Results. All statistical tests were two tailed and carried out in Statistica v6.1 and SPSS v22 (IBM SPSS Statistics for Windows, IBM Corporation, Armonk, NY, U.S.A.).

\section{Video analysis}

The first field trip in March 2012 served as a pilot study to train the experimenter and assistants and to test the number of possible playback trials per individual. In addition, we obtained a set of a priori behaviours. We focused on behaviours that are informative in the detection of differences in the response of the bats (only slightly modified during video analysis if a bat showed a new behaviour). These behaviours allowed us to quantify (occurrence, count data) and qualify (duration) each subject's response to the stimuli. The behavioural variables (listed and defined in Table A2 in the Appendix) were classified a posteriori into the main categories inactive/calm, active and attentive behaviours. Attentive behaviours are a subset of active behaviours. We defined attentive behaviours as behaviours that imply a stimulus-directed response of the bat to the playbacks by either orienting itself towards the sound source (e.g. head raised), by revealing a listening response (e.g. ears twitching) or by showing a startle response (cringing as indicated by leg contraction). Active behaviours, on the other hand, which are rated nonattentive comprise movements of the bat that occur when it settles down (e.g. scrambling) and do not indicate any reaction to the acoustic stimulus (e.g. wing stretching). This classification was verified throughout the video analysis and categorized by three different observers.

Each trial was analysed frame by frame with 25 frames/s using the software InterAct (InterAct 8.0, Mangold International). We restricted the analysis to three $15 \mathrm{~s}$ time periods (Fig. 3 ): the first $15 \mathrm{~s}$ of the habituation (HabSTART), the last $15 \mathrm{~s}$ of the habituation (HabEND) and the $15 \mathrm{~s}$ long test stimuli (TEST) ending with the motivational control stimulus (MOT) and the synchronization signal (SYNC).

Two observers (A.B. and D.S.J.) coded the behaviours of bats in the videos of the trials. In all cases, the researchers had no knowledge about the stimuli class used in the videos. To ensure consistency in video coding, 10 randomly chosen trials were double coded by both researchers and tested for interobserver reliability. We used Cohen's kappa for count data (Interact v8.0 electronic manual, Mangold International) and Kendall's tau to test the durations of the behaviours for congruence between the observers. Finally, we tested whether females and males differed in their response behaviour by comparing the duration of active behaviours with a Mann-Whitney $U$ test.

Trials were regarded as invalid and thus not analysed if the data for an individual were incomplete, if a bat did not react to the initial habituation playback, if the bat left the perch during the final phase of habituation and/or the test playback or if the pause caused by switching between playbacks interrupted the habituated state. We verified that bats were still habituated by comparing the duration of attentive behaviours during HabEND versus the last series of postgap habituation calls (PGH, see Fig. 3) using Wilcoxon matched-pairs tests. We controlled for false positives by using the control test playback, which contained calls of the same population used in the habituation file, to assess the frequency of occurrence of spontaneous recoveries from habituation, i.e. a false positive response, We controlled for false negatives, i.e. the failure of the bat to react to a test stimulus because of sensory/experimental fatigue (Pancratz \& Cohen, 1970), by determining whether the bat responded to the motivational control. We tested whether bats were habituated before playing back the test stimulus by comparing the occurrence of behaviours at the beginning and end of the habituation phase as well as the duration of attentive behaviours. Each trial was tested using chi-square tests in which the expected number of frames for 'inactive/calm' and 'attentive' for HabEND was the number of frames counted in the HabSTART phase and compared to the observed number of frames counted during HabEND. The duration of attentive behaviours during HabSTART and HabEND was tested across all trials using a Wilcoxon matchedpairs test. The effects of long-term habituation on the general level of responsiveness of the bats needed to be evaluated because the experiment was done over several days. This was done by comparing the duration of attentive behaviours for the beginning of the habituation playback across the experimental nights using a Friedman ANOVA. After confirming the validity of the trials we tested discrimination between the bat's own population echolocation calls versus the different classes of test playback echolocation calls by comparing the duration of attentive behaviours during the end of the habituation phase (HabEND) and during test playbacks (TEST). We did this comparison for each playback category across individuals using Wilcoxon matched-pairs test.

\section{Audio analysis}

Large audio files were split using Wave Splitter v2.10 to obtain a manageable file size. Corrupted wav files were recovered using Audacity v2.0.3 (Audacity Team, SourceForge.net).

Acoustic measurements (Fig. 4) were taken on calls of each bat and of the playback stimuli using the automatic measurement function in Avisoft SASLab Pro v5.2. We analysed 10-30 calls of each experimental individual from recordings obtained during experiments. We chose calls of similarly good quality at the beginning of the trial before any playback was started and the bats showed orientation behaviour in the box, such as scanning the environment. We, again, chose calls emitted after the first 10 calls, avoiding lower frequency calls of the tuning in phase (the first series of calls emitted after a silent period show lower RFs and gradually 'tuned in' to the individuals' typical RFs; Siemers et al., 2005).

We measured the duration of each call, the interval between the onsets of two calls, the distance from the beginning of each call until it reached its maximum energy, the peak frequency of each call, the frequency of the first and third harmonics, the minimum frequency of the frequency-modulated components at the beginning and at the end of the prominent second harmonic and the call's entropy which quantifies the pureness of sounds (its value is zero for pure-tone signals and one for random noise; see AvisoftSASLab Pro manual v5.1, p. 162).

\section{RESULTS}

In total we obtained complete and analysable data for 36 bats: 15 (seven males; eight females) were from Lekkersing and 21 (10 males; 11 females) from De Hoop. 


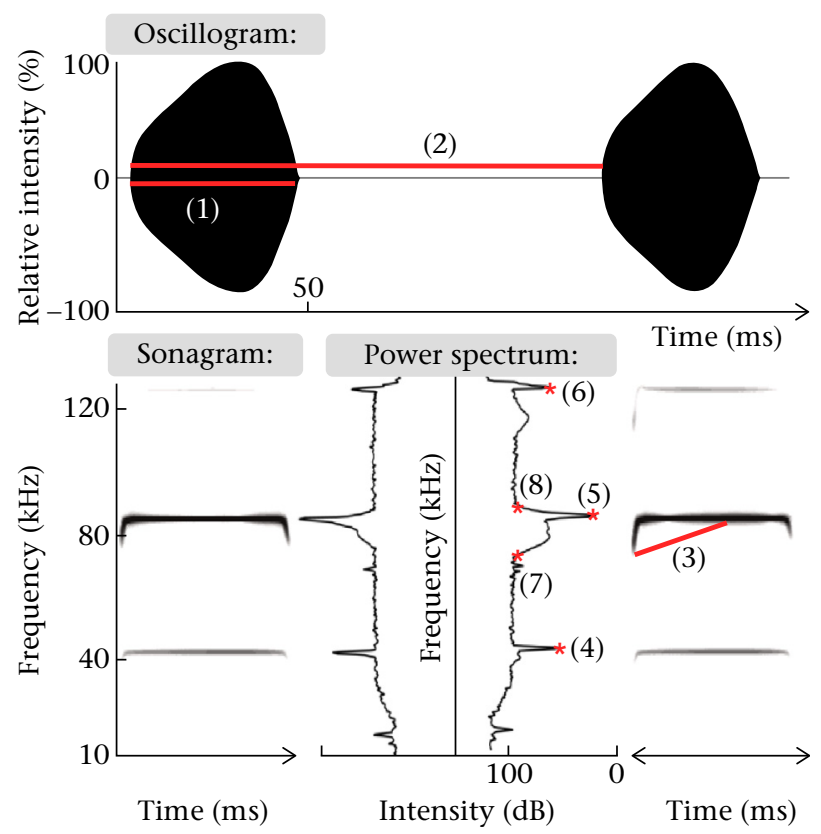

Figure 4. Example of two R. capensis echolocation calls. The parameters measured for the acoustic analysis are indicated in the oscillogram, the sonagram or the power spectrum. Lines indicate duration measurements, asterisks indicate points of measurements and numbers next to asterisks refer to each parameter: (1) call duration, (2) interval, (3) distance to maximum amplitude, (4) frequency fundamental harmonic, (5) peak frequency second harmonic (RF), (6) frequency third harmonic, (7) minimum frequency at start (second harmonic), (8) minimum frequency at end (second harmonic).

The interobserver reliability test indicated high conformity between the observers (Cohen's kappa coefficient: $\kappa>0.77, N=10$, $P<0.05$ ) as well as concordance of duration of behaviours (Kendal's tau rank correlation coefficient: $\tau=0.7, Z=3.580, P<0.001)$. We found no difference in the response behaviour between males and females and therefore grouped all bats for each sample site (Mann-Whitney $U$ test: RcaLS75 bats: $U=625.0, Z_{\text {adjusted }}=-0.78$, $N_{\text {males }}=7, \quad N_{\text {females }}=8, P=0.431 ;$ RcaDH85 bats: $U=1209.5$, $\left.Z=1.32, N_{\text {male }}=10, N_{\text {female }}=11, P=0.188\right)$. We observed a moderate and nonsignificant decline in responsiveness over experimental days (Friedman ANOVA: RcaLK75 data set: $\chi^{2}{ }_{14}=1.200$, $P=0.549$; RcaDH85 data set: $\chi_{20}^{2}=10.987, P=0.052$ ). Our tests confirmed a successful habituation at the end of the habituation phase for each trial (chi-square test: $\chi^{2}{ }_{1} \geq 3.84, P \leq 0.05$, single tests not shown). The duration of attentive behaviours also confirmed a decrease in active behaviours from the beginning to the end of the habituation phase across individuals per playback class (see next section; Wilcoxon matched-pairs test: RcaLS75 data set: $T=39.50, N=75, P<0.01 ;$ RcaDH85 data set: $T=84.50$, $N=126, P<0.001)$.

\section{Discrimination Task}

The bats from Lekkersing, RcaLS75, showed a reaction by becoming active again in 51 of 75 trials (68\%). In trials with the test playback RcaDH85, RcaTF86 or the sympatric Rda85, all 15 bats showed a reaction in all trials (Fig. 5a). In one trial of the control playback RcaLS75 one bat showed a reaction (false positive). In this case the reaction was a nonattentive active behaviour. When habituated with the calls of the other phonetic populations, RcaDH85, and tested against the sympatric Rda85, bats showed a reaction in five of 15 trials. Overall, this is a significant difference between the playback classes (Cochran's $Q$ test: $Q_{4}=46.359$, $N=15, P<0.001)$.
The bats from De Hoop, RcaDH85, reacted in 85 of 126 trials (67.5\%) with active behaviours. Twice the bats reacted with nonattentive active behaviours to the control playback RcaDH85 (Fig. 5b). In trials with test playback calls of the sympatric $R$. clivosus, Rcl92, all bats reacted and in all but one trial bats reacted when we played the allopatric $R$. damarensis, Rda85, and RcaLS75 echolocation calls. When hearing echolocation calls from conspecifics using slightly different resting peak frequencies, RcaHH84 and RcaTF86, the bats reacted ambiguously: 10 reacted to calls from RcaHH84 bats and 12 to calls from RcaTF86 bats. Here, as with the RcaLS75 bats, we found a significant difference between the responses to the different playback classes (Cochran $Q$ test: $Q_{5}=53.808, N=21, P<0.001$ ).

The bats from Lekkersing, RcaLS75, showed a dishabituation response to the playbacks of RcaDH85, RcaTF86 and the sympatric species Rda85 when habituated to their own population calls RcaLS75 (Wilcoxon matched-pairs test: $T=0.00, Z=0.41, N=15$, $P=0.001 ; T=0.00, Z=3.42, P=0.001 ; T=19.0, Z=0.85, N=15$, $P=0.004$; Fig. 6a). However, when previously habituated to calls from the other phonetic population, RcaDH85, they showed no attentive reaction to the calls of the sympatric species Rda85. They also showed no significant reaction to calls from their own population (i.e. no false positive; Fig. 6a).

When habituated with their own calls, the bats from De Hoop in the south, RcaDH85, mostly did not discriminate between calls from acoustically similar phonetic populations of their own species, RcaHH84 and RcaTF86, but did show a significant response to the acoustically dissimilar population RcaLS75 and the other two species Rda85 and Rcl92 although only one of them, Rcl92, co-occurs with them at De Hoop (Wilcoxon matched-pairs test: $T=1.0$, $Z=3.88, N=21, P<0.001 ; T=4.0, Z=3.77, N=21, P<0.001$; $T=0.0, Z=4.02, N=21, P<0.001$; Fig. $6 \mathrm{~b}$ ). They did not react to the playbacks of the control calls of their own population (i.e. no false positive; Fig. 6b).

The difference in discriminatory ability between the two test populations is also apparent when comparing the response strength in relation to the acoustic similarity of the playbacks (Fig. 7). The playback from the phonetic population RcaLS75 is acoustically dissimilar to the other playbacks based on the first two functions of a DFA which predominantly consisted of frequency and temporal parameters (see Fig. 2 and section Stimulus Generation in the Methods for details). Bats from this population showed strong responses towards all playbacks from other species or conspecific phonetic populations (red, green and grey columns on the left in Fig. 7a) and low response levels towards its own calls (control, grey column on the right in Fig. 7a). Accordingly, the bats from the phonetic population RcaDH85 showed a strong response towards the acoustically dissimilar playback of the conspecific phonetic population RcaLS75 (grey column on the right in Fig. 7a), but an almost similarly strong response to the acoustically similar playback of the allopatric heterospecific Rda85 (green column in Fig. 7a). Correspondingly, the relationship between acoustic distance and response strength was stronger for the bats from Lekkersing RcaLS75 (Fig. 7b) and weaker for the bats from De Hoop RcaDH85 (Fig. 7c). Although the acoustic distance of Rda85 calls to echolocation calls of RcaDH85 was low, the response strength of RcaDH85 bats was high towards the playbacks of Rda85 (greyish area in the graphs). The respective narrow area of acoustic distance (as set by the variation of calls used by conspecifics of the listening bats) for the RcaLS75 bats shows lower response levels towards playback calls inside this area. The increased response strength showed that bats from De Hoop discriminated between calls from their own population (RcaDH85) and calls from the allopatric heterospecific (Rda85), despite overlapping frequencies and general acoustic similarity. In contrast, bats from Lekkersing showed no 

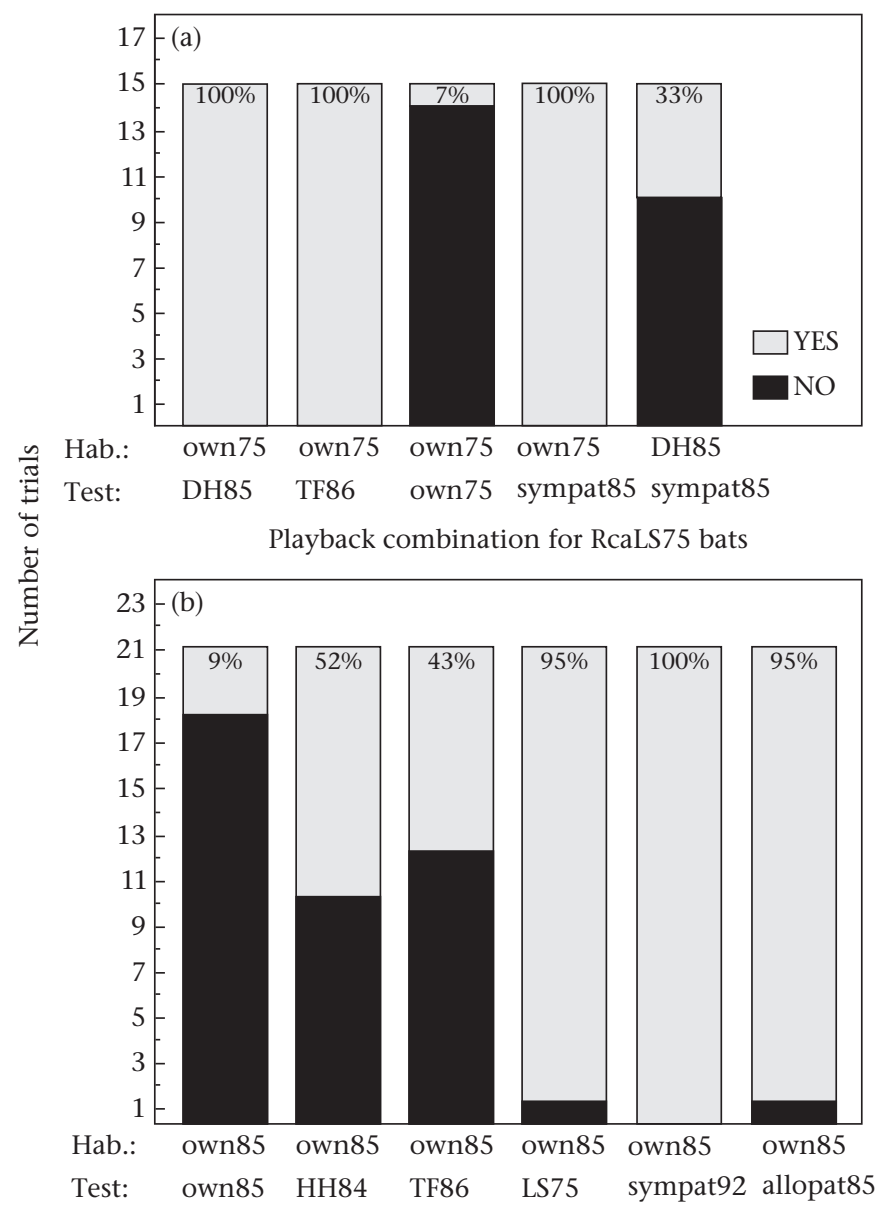

Playback combination for RcaDH85 bats

Figure 5. Number of trials in which the bats from the two study sites (a) LS and (b) DH discriminated (yes) or did not discriminate (no) the calls of the test playback from the calls in the preceding habituation playback by showing activity.

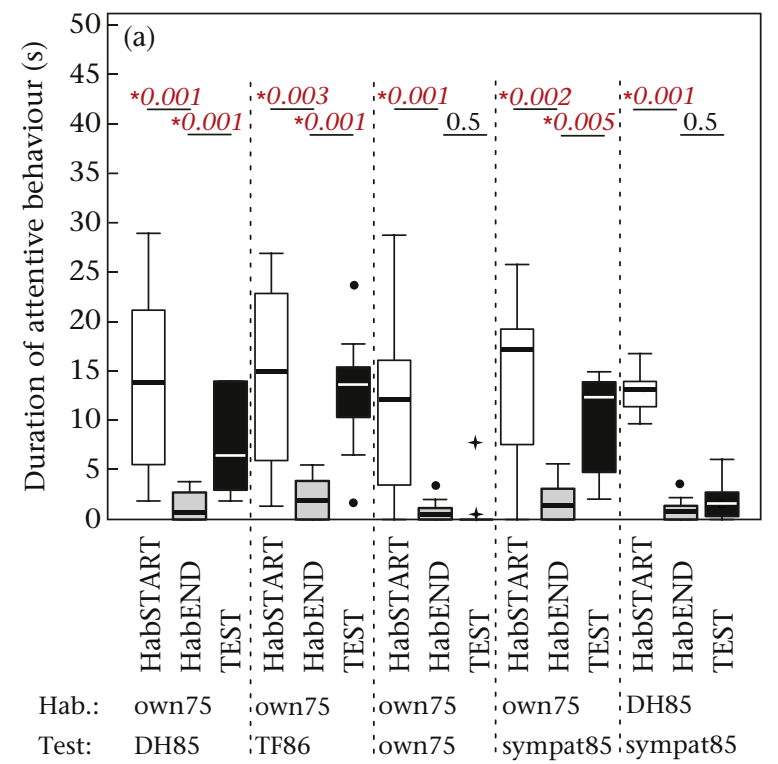

Playback combination for RcaLS75 bats increased response, i.e. no discrimination between these two similar echolocation playbacks (RcaDH85 and Rda85) compared to the control (own population calls).

\section{DISCUSSION}

In both test populations, $R$. capensis bats were able to discriminate between their own calls and calls of the respective sympatric horseshoe bat species. Furthermore, the echolocation calls of bats from Lekkersing were perceived as distinctly different from those of all other phonetic populations of $R$. capensis by the bats from both phonetic populations. However, our results suggest that the bats from the two test populations differed in their ability to discriminate between calls of conspecifics and heterospecifics of the same resting peak frequency. De Hoop bats displayed this ability but Lekkersing bats did not. The prediction of the $\mathrm{ACH}$ that increased signal overlap between calls of sympatric species should increase the rates of misclassification of calls from conspecifics or heterospecifics was therefore confirmed for bats from Lekkersing. However, bats from De Hoop were able to discriminate between species using overlapping RFs. This confirms that bats are able to use additional acoustic cues besides frequency to discriminate conspecifics from heterospecifics.

This study also provides evidence that overlapping signals among species occurring in the same habitat can be accommodated by the evolution of increased perceptual acuity in the receiver. Although this has been shown in other taxa (e.g. birds: Seddon \& Tobias, 2010; Tobias et al., 2014; frogs: Amézquita et al., 2006; 2011; Marshall et al., 2006; insects: Nosil et al., 2003; Jang \& Gerhardt 2006) to our knowledge this is the first time it has been shown in a mammal. It is also the first observation in any mammal of the evolution of differences in perceptual acuity within the same species mediated by differences in the complexity of signalling assemblages and constraints imposed by the dual function of the trait (see below). This observation suggests that the availability of signal space for a particular species may mediate the evolutionary response of this species to competition for a unique communication channel from sympatric species. Signal space is the range of

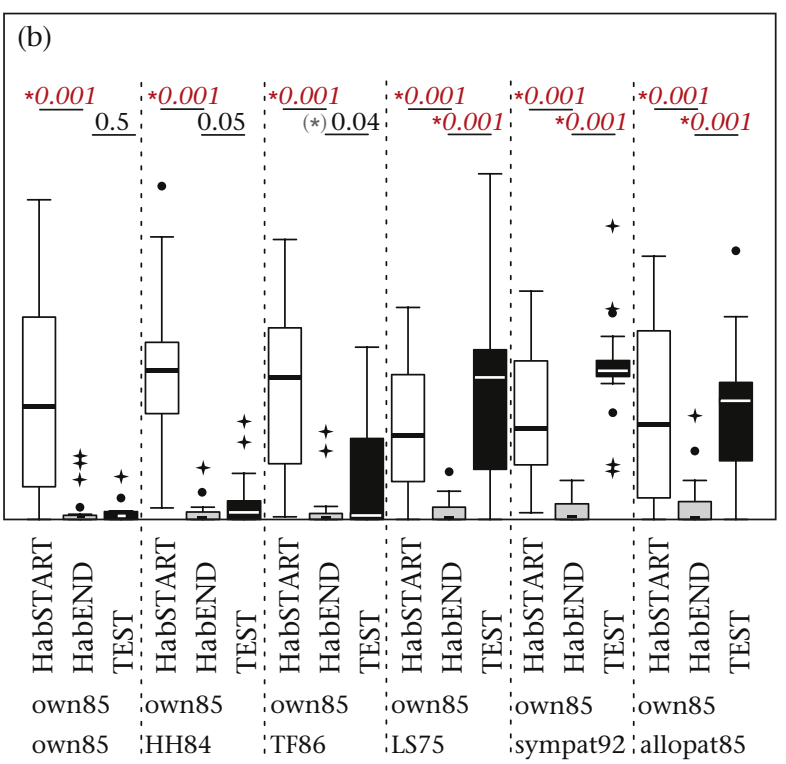

Playback combination for RcaDH85 bats

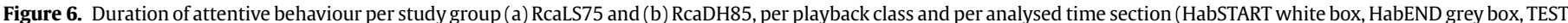

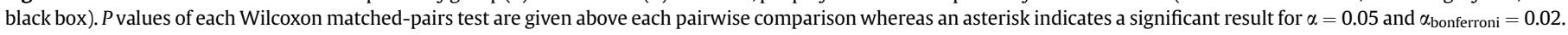

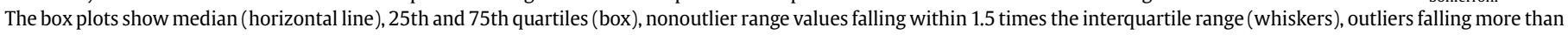
1.5 times the interquartile range from the edge of the box (circles) and extremes falling more than 2.0 times the interquartile range from the edge of the box (crosses). 

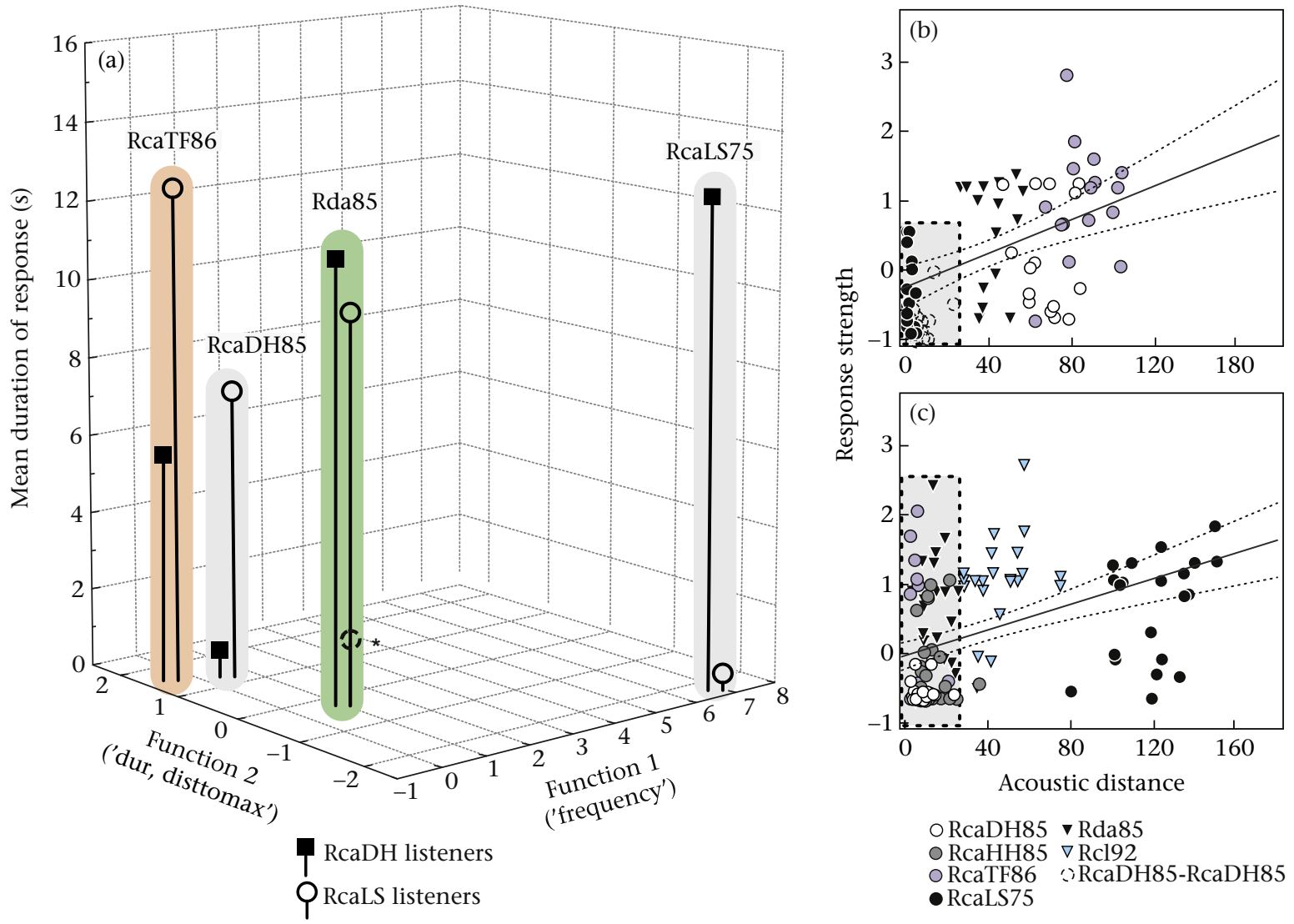

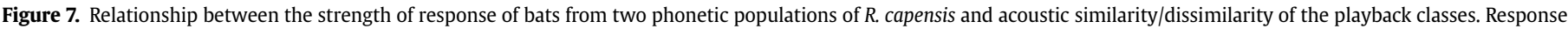

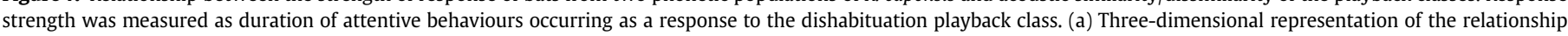

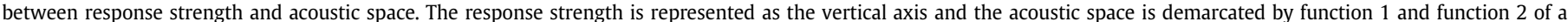

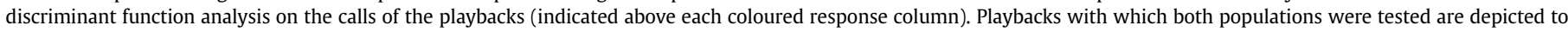

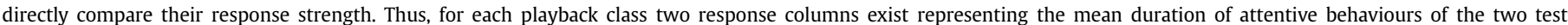

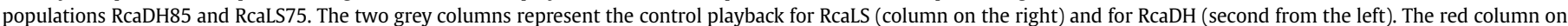

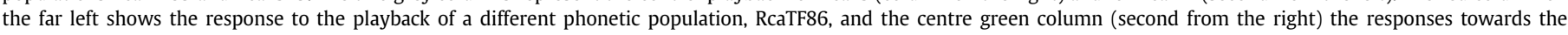

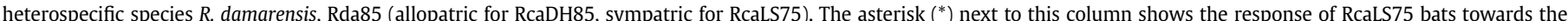

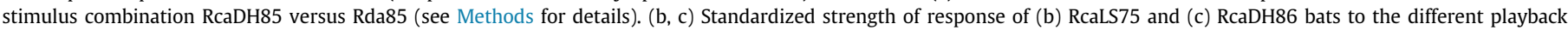

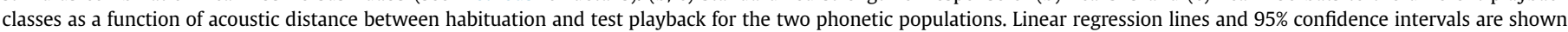

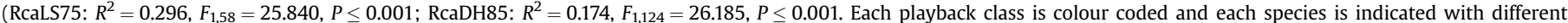
symbols (see key below graph).

acoustic signals theoretically available to a species into which its signal could diverge and is delimited by sympatric species but also by constraints imposed by the dual function of a sensory system. Thus, where signal space permits, potential misclassification of signals as a result of overlapping signals used by sympatric species may be minimized through the evolution of signal divergence through character displacement. However, where signal space is limited the evolution of increased perceptual acuity among receivers may allow both coexistence and convergence of signals (see also Seddon \& Tobias, 2010).

The use of multiple cues in acoustic signals for vocal recognition of kin, mates or competitors is not a novel discovery and is well established for birds, anurans and mammals (mammals: Hammerschmidt \& Todt, 1995; anurans: Vignal \& Kelley, 2007; birds: Vignal, Mathevon, \& Mottin, 2008) although species discrimination based on multiple acoustic cues in echolocating animals was only suspected for bats (Schuchmann et al., 2012). The ACH, as currently applied to bats (e.g. Heller \& von Helversen, 1989; Jacobs et al., 2007), is thus too narrow in its formulation as it is based on resting frequency alone. It should be expanded to incorporate multiple call components as was initially done for anurans (Duellman \& Pyles, 1983 ) so that it more accurately describes the situation in bats and is more broadly applicable to other taxa. We suggest the following formulation of the hypothesis. In an assemblage of sympatric heterospecific animals using acoustic signals, multidimensional acoustic space is partitioned in such a way that each species occupies a distinct acoustic space facilitating intraspecific communication and species discrimination. This would also distinguish the ACH from the spectral partitioning hypothesis (Amézquita et al., 2011).

\section{Local Acoustic Signalling Assemblages and Perceptual Acuity}

The fact that the bats from the two study sites differed in their ability to use additional acoustic cues in echolocation calls might be a result of differences in the local acoustic assemblages. Lekkersing is remote and bats there are isolated from other $R$. capensis populations as evidenced by limited historical gene flow between Lekkersing and other populations (Odendaal et al., 2014). Rhinolophus capensis at Lekkersing are therefore only exposed to the calls of a single different rhinolophid species and were unable to discriminate between conspecific and heterospecific (R. damarensis) calls that were similar but different to its own. This means that they may use a simple rule of thumb that bats calling at different frequencies are heterospecifics. This rule of thumb results 
in their being unable to distinguish between heterospecifics and conspecifics echolocating at similar and higher frequencies. In contrast, the acoustic assemblage for bats from De Hoop is more complex than that encountered by $R$. capensis at Lekkersing. At De Hoop and the region around it there are populations of bats of the same species with slightly different RFs in close geographical proximity. These bats are thus likely to be exposed to a higher variation of frequencies within their own species and the frequent gene flow between these populations in the past (Odendaal et al., 2014) suggests an ability to identify individuals from these phonetic populations as conspecifics. The acoustic challenge for these bats is further complicated by the co-occurrence of the heterospecific $R$. clivosus which has a frequency range overlapping, albeit slightly, with that of its own. Finer levels of species discrimination and conspecific recognition based on frequency, as well as other acoustic cues coded in echolocation calls, may therefore provide a selective advantage. This interpretation of our data is further supported by the ability of $R$. capensis at De Hoop to clearly discriminate between calls of their own species and those from an allopatric heterospecific, $R$. damarensis, using the same resting frequency. We therefore propose that adaptation of the receiver's sensory systems to the local acoustic assemblage may have resulted in a hierarchy of discrimination abilities within the same species. Simple rules of thumb based on divergent call frequencies alone may provide sufficient selective advantage in a simple acoustic assemblage with more available signal space, such as that experienced by $R$. capensis at Lekkersing, but additional acoustic cues to discriminate species are necessary when bats live in a more complex acoustic assemblage with less available signal space.

\section{Perceptual Acuity and Evolutionary Constraints}

Communication signals, especially those with dual functions, may be precluded from reaching their optimum character states as a result of evolutionary constraints. The primary functions of echolocation are orientation and food acquisition. Encoding unique communication cues in echolocation may have an adverse impact on these functions. However, an information-carrying signal that is produced almost constantly and is freely available is a likely candidate to be used for communication. Signals emitted by an animal, voluntarily or involuntarily, provide useful information, for example about feeding grounds and suitable roosts (Barclay, 1982; Ruczynski, Kalko, \& Siemers, 2007), the presence of mates (Behr \& Von Helversen, 2004; Leippert, 1994), prey (Page, Ryan, \& Bernal, 2013; Siemers, Kriner, Kaipf, Simon, \& Greif, 2012), a threat (Mariappan, Bogdanowicz, Marimuthu, \& Rajan, 2013; Russ, Jones, Mackie, \& Racey, 2004) or attributes of the sender (Fenton, 2003; Siemers et al., 2005) and its behavioural state (Bastian \& Schmidt, 2008). Nevertheless, the communicative function of echolocation can only arise or be fine-tuned if it does not compromise the primary functions of echolocation which are fundamental to the bat's survival. This might explain why there appears to be so little partitioning of sonar frequency bands at De Hoop, contrary to the $\mathrm{ACH}$, whereas at Lekkersing the anomalously low calls of $R$. capensis may have evolved because it avoids overlap with $R$. damarensis. At Lekkersing selection that optimizes echolocation for orientation and food acquisition and selection that allows more effective communication operate in the same direction, i.e. lowering call frequency. Lower frequency calls increase prey detection distances in the relatively open habitat at Lekkersing (Odendaal et al., 2014) and avoid overlap with $R$. damarensis. In contrast, the dense vegetation within which these bats forage at De Hoop probably selects for higher frequencies (Odendaal et al., 2014) whereas advantages that accrue through more effective communication as a result of nonoverlapping call frequencies with $R$. clivosus would select for lower frequencies. Avoiding call overlap with $R$. clivosus by using higher frequency calls would probably also require a reduction in body size for increased flight manoeuvrability (Norberg \& Rayner, 1987) to deal with the decreased detection range resulting from increased atmospheric attenuation of higher frequency calls (Lawrence \& Simmons, 1982; Schnitzler \& Kalko, 2001). All rhinolophids echolocating at higher frequencies than $R$. clivosus (e.g. Rhinolophus swinnyi and Rhinolophus denti) are much smaller than R. capensis (Monadjem et al., 2010). At De Hoop there may thus be a trade-off between the orientation/food acquisition functions of echolocation and its communicative role, i.e. selection to optimize these functions is exerted in opposite directions. The costs to the orientation and food acquisition functions of echolocation may have precluded the evolution of call frequencies in $R$. capensis that were divergent from those of $R$. clivosus. Instead, communication via echolocation at De Hoop may have been optimized through the evolution of finer levels of discrimination based on other components of the call. This is similar to the evolution of overlapping male songs across several congeneric species in a complex multispecies community of forest-dwelling birds (Tobias et al., 2014; Tobias \& Seddon, 2009). This overlap was apparently driven by the dual function of birdsong in mate attraction and interspecific territory defence in shared habitats. Although mate attraction requires unique, individualized male song, territory defence selects for convergence in male song among different species allowing them to function well in advertising the male's territory across species. Overlapping male songs have, however, resulted in the evolution of increased perceptual acuity among female receivers allowing the continued functioning of birdsong in intraspecific communication (Tobias et al., 2014). In this example increased perceptual acuity is driven by constraints imposed by the dual function of birdsong in communication whereas in our study it is driven by constraints imposed by selection for noncommunicative functions, i.e. orientation and food acquisition. The validity of the $\mathrm{ACH}$ as an explanation for call frequency divergence within a species is therefore tempered by the different selection pressures within the local acoustic assemblage that each population may experience. The model system of $R$. capensis thus provides a novel example of the evolution of mammalian communication systems by revealing the receiver's adaptation to evolve or maintain species-specific communication.

\section{Conclusions}

Species discrimination ability, even within the same species, is dependent on local acoustic signalling assemblages that select for the ability to discriminate and may involve a trade-off between the different functions of a multifunctional trait. Acoustic species discrimination can be achieved by the partitioning of signal space via divergence of the sender's calls or through increased perception abilities of receivers.

\section{Acknowledgments}

We specifically thank Lizelle J. Odendaal for her contribution in collecting echolocation calls of $R$. capensis, for contributing to the preparation of playback files, and for partially doing the experiments with us. We also thank Björn Siemers and Robert Barclay for crucial comments on the experimental design used in this study and practical advice during our first field trip. Echolocation data were collected by Hassan Babiker, Megan Cunnama and Orsilla Schmit. We had great help in capturing and caring for bats during the experiments from Tinyiko Maluleke, Gregory Mutumi and Robert Raw. We thank the editors and two anonymous referees for their constructive comments, which helped us to improve the 
manuscript. This study was financially funded by research grants to D.S.J. from the South African Research Chair Initiative of the Department of Science and Technology and administered by the National Research Foundation (NRF) of South Africa (GUN 64798), as well as by postdoctoral research grants to A.B. (URC Postdoctoral research grant of UCT (URC-BSTANN006) and NRF-freestanding postdoctoral fellowship for Non-South Africans (88359)).

\section{References}

Altringham, J. D., \& Fenton, M. B. (2003). Sensory ecology and communication in the Chiroptera. In T. H. Kunz, \& M. B. Fenton (Eds.), Bat ecology (pp. 90-118). Chicaco, IL: The University of Chicago Press.

Amézquita, A., Hödl, W., Lima, A. P., Castellanos, L., Erdtmann, L., \& De Araújo, M. C. (2006). Masking Interference and the evolution of the acoustic communication system in the Amazonian dendrobatid frog Allobates femoralis. Evolution, 60(9), 1874-1887.

Amézquita, A., Flechas, S. V., Lima, A. P., Gasser, H., \& Hödl, W. (2011). Acoustic interference and recognition space within a complex assemblage of dendrobatid frogs. Proceedings of the National Academy of Sciences of the United States of America, 108(41), 17058-17063.

Anderson, M. J., Ambrose, L., Bearder, S. K., Dixon, A. F., \& Pullen, S. (2000). Intraspecific variation in the vocalizations and hand pad morphology of southern lesser bush babies (Galago moholi): a comparison with G. senegalensis. Inter national Journal of Primatology, 21(3), 537-555.

Barclay, R. M. R. (1982). Interindividual use of echolocation calls: eavesdropping by bats. Behavioral Ecology and Sociobiology, 10(4), 271-275.

Bastian, A., \& Schmidt, S. (2008). Affect cues in vocalizations of the bat, Megaderma lyra, during agonistic interactions. Journal of the Acoustical Society of America, 124(1), 598-608.

Behr, O., \& Von Helversen, O. (2004). Bat serenades-complex courtship songs of the sac-winged bat (Saccopterix bilineata). Behavioral Ecology and Sociobiology, 56 $106-115$.

Bouton, M. E. (2007). Learning and behavior: A contemporary synthesis. Sunderland, MA: Sinauer Associates.

Bradbury, J., \& Vehrencamp, S. (2011). Principles of animal communication. Sunderland, MA: Sinauer Associates.

Braune, P., Schmidt, S., \& Zimmermann, E. (2008). Acoustic divergence in the communication of cryptic species of nocturnal primates (Microcebus ssp.). BMC Biology, 6(1), 1-19.

Brinkløv, S., Fenton, M. B., \& Ratcliffe, J. M. (2013). Echolocation in oilbirds and swiftlets. Frontiers in Physiology, 4, 1-12.

Bukatko, D., \& Daehler, M. W. (2003). Child development: A thematic approach (5th ed.). Boston, MA: Houghton Mifflin.

Catchpole, C. K., \& Slater, P. J. B. (2008). Bird song: biological themes and variations Cambridge, U.K.: Cambridge University Press.

Charlton, B. D., Huang, Y., \& Swaisgood, R. R. (2009). Vocal discrimination of potential mates by female giant pandas (Ailuropoda melanoleuca). Biology Letters, 5(5), 597-599.

Cowen, R. (2005). History of life (4th ed.). Oxford, U.K.: Blackwell Publishing.

Dechmann, D. K. N., Wikelski, M., Van Noordwijk, H. J., Voigt, C. C., \& VoigtHeucke, S. L. (2013). Metabolic costs of bat echolocation in a non-foraging context support a role in communication. Frontiers in Physiology, 4, 1-6.

Denzinger, A., \& Schnitzler, H.-U. (2013). Bat guilds, a concept to classify the highly diverse foraging and echolocation behaviors of microchiropteran bats. Frontiers in Physiology, 4, 1-15.

Dorado Correa, A. M., Goerlitz, H. R., \& Siemers, B. M. (2013). Interspecific acoustic recognition in two European bat communities. Frontiers in Physiology, 4, 1-8.

Duellman, W. E., \& Pyles, R. A. (1983). Acoustic resource partitioning in anuran communities. Copeia, 1983, 639-649.

Eimas, P. D., Siqueland, E. R., Jusczyk, P., \& Vigorito, J. (1971). Speech perception in infants. Science, 171(3968), 303-306.

Fantz, R. L. (1964). Visual experience in infants: decreased attention to familiar patterns relative to novel ones. Science, 146(3644), 668-670.

Fenton, M. B. (1985). Communication in the Chiroptera. Bloomington, IN: Indiana University Press.

Fenton, M. B. (2003). Eavesdropping on the echolocation and social calls of bats Mammal Review, 33(3-4), 193-204.

Fitch, T. W. (2006). Production of vocalizations in mammals. In K. Brown (Ed.) Encyclopedia of language and linguistics (pp. 115-121). Oxford, U.K.: Elsevier.

Grant, B. R., \& Grant, P. R. (2010). Songs of Darwin's finches diverge when a new species enters the community. Proceedings of the National Academy of Sciences of the United States of America, 107(47), 20156-20163.

Gregg, J. D., Dudzinski, K. M., \& Smith, H. V. (2007). Do dolphins eavesdrop on the echolocation signals of conspecifics? International Journal of Comparative Psychology, 20(1), 65-88.

Guillen, J. (2012). FELASA guidelines and recommendations. Journal of the American Association for Laboratory Animal Science, 51(3), 311-321.

Guillén, A., Juste, J. B., \& Ibañez, C. (2000). Variation in the frequency of the echolocation calls of Hipposideros ruber in the Gulf of Guinea: an exploration of the adaptive meaning of the constant frequency value in rhinolophoid CF bats. Journal of Evolutionary Biology, 13, 70-79.
Hammerschmidt, K., \& Todt, D. (1995). Individual differences in vocalisations of young barbary macaques (Macaca sylvanus): a multi-parametric analysis to identify critical cues in acoustic signalling. Behaviour, 132(5/6), 381-399.

Heller, K.-G., \& von Helversen, O. (1989). Resource partitioning of sonar frequency bands in rhinolophoid bats. Oecologia, 80(2), 178-186.

Jacobs, D. S. (n.d.) [Audio recordings of juvenile Rhinolophus clivosus.] Unpublished raw data.

Jacobs, D. S., Babiker, H., Bastian, A., Kearney, T., van Eeden, R., \& Bishop, J. M. (2013). Phenotypic convergence in genetically distinct lineages of a Rhinolophus species complex (Mammalia, Chiroptera). PLoS One, 8(12), e82614.

Jacobs, D. S., Barclay, R. M. R., \& Walker, M. (2007). The allometry of echolocation call frequencies of insectivorous bats: why do some species deviate from the pattern? Oecologia, 152, 583-594.

Jang, Y., \& Gerhardt, H. C. (2006). Divergence in female calling song discrimination between sympatric and allopatric populations of the southern wood cricket Gryllus fultoni (Orthoptera: Gryllidae). Behavioral Ecology and Sociobiology, $60(2), 150-158$

Jones, G. (1997). Acoustic signals and speciation: the roles of natural selection and sexual selection in the evolution of cryptic species. Advances in the Study of Behavior, 26, 343-344.

Jones, G. (1999). Scaling of echolocation call parameters in bats. Journal of Experimental Biology, 202, 3359-3366.

Jones, G., \& Siemers, B. M. (2010). The communicative potential of bat echolocation pulses. Journal of Comparative Physiology A, 197(5), 447-457.

Jones, G., \& Van Parijs, S. M. (1993). Bimodal echolocation in pipistrelle bats: are cryptic species present? Proceedings of the Royal Society B: Biological Sciences, $251,119-125$.

Kingston, T., Jones, G., Zubaid, A., \& Kunz, T. H. (2000). Resource partitioning in rhinolophoid bats revisited. Oecologia, 124(3), 332-342.

Kingston, T., Lara, M. C., Jones, G., Akbar, Z., Kunz, T. H., \& Schneider, C. J. (2001). Acoustic divergence in two cryptic Hipposideros species: a role for social selection? Proceedings of the Royal Society B: Biological Sciences, 268(1474), 1381-1386.

Kingston, T., \& Rossiter, S. J. (2004). Harmonic-hopping in Wallacea's bats. Nature, 429(6992), 654-657.

Knörnschild, M., Jung, K., Nagy, M., Metz, M., \& Kalko, E. (2012). Bat echolocation calls facilitate social communication. Proceedings of the Royal Society B: Biological Sciences, 279(1748), 4827-4835.

Kulzer, E. (2005). Chiroptera. In Handbuch der zoologie [. In Handbook of zoology] (Vol. 3). Berlin, Germany: Walter de Gruyter.

Lawrence, B. D. \& Simmons, J. A. (1982). Measurements of atmospheric attenuation at ultrasound frequencies and the significance for echolocation by bats. Journal of the Acoustical Society of America, 71, 585-590.

Leippert, D. (1994). Social behaviour on the wing in the false vampire. Megaderma lyra. Ethology, 98(2), 111-127.

Liebermann, P., \& Blumstein, S. E. (1991). Speech physiology, speech perception, and acoustic phonetics. Camebridge, U.K.: Camebridge University Press.

Li, Y., Wang, J., Metzner, W. Luo, B., Jiang, T. Yang, S., et al. (2013). Behavioral responses to echolocation calls from sympatric heterospecific bats: implications for interspecific competition. Behavioral Ecology and Sociobiology, 68(4), 657-667.

Maltby, A., Jones, K. E., \& Jones, G. (2010). Chapter 2.4-Understanding the evolutionary origin and diversification of bat echolocation calls. In M. B. Stefan (Ed.), Handbook of mammalian vocalizations (Vol. 19, pp. 37-47). Oxford, U.K.: Academic Press.

Mariappan, S., Bogdanowicz, W., Marimuthu, G., \& Rajan, K. E. (2013). Distress calls of the greater short-nosed fruit bat Cynopterus sphinx activate hypothalamicpituitary-adrenal (HPA) axis in conspecifics. Journal of Comparative Physiology A, 199(9), 775-783.

Marshall, V. T., Schwartz, J. J., \& Gerhardt, H. C. (2006). Effects of heterospecific call overlap on the phonotactic behaviour of grey treefrogs. Animal Behaviour, 72(2), 449-459.

Maynard Smith, J., \& Harper, D. (2003). Animal signals. Oxford, U.K.: Oxford University Press.

Mc Gregor, P. K., Falls, B. I., Catchpole, C. K., Pepperberg, I. M., Gerhardt, C. H., et al. (1992). Playback and studies of animal communication. New York, NY: Springer.

Mendelson, T. C., \& Shaw, K. L. (2012). The (mis)concept of species recognition. Trends in Ecology \& Evolution, 27(8), 421-427.

Monadjem, A., Taylor, P. J., Cotterill, W., \& Schoeman, M. C. (2010). Bats of southern and central Africa: a biogeographic and taxonomic synthesis. Johannesburg, South Africa: Wits University Press.

Monteiro, A., \& Podlaha, O. (2009). Wings, horns, and butterfly eyespots: how do complex traits evolve? PLoS Biology, 7(2), e1000037.

Neuweiler, G. (1990). Auditory adaptations for prey capture in echolocating bats. Physiological Reviews, 70(3), 615-641.

Neuweiler, G., Bruns, V., \& Schuller, G. (1980). Ears adapted for the detections of motion, or how echolocating bats have exploited the capacities of the mammalian auditory system. Journal of the Acoustical Society of America, 68(3), $741-753$.

Neuweiler, G., Metzner, W., Heilmann, U., Rübsamen, R., Eckrich, M., \& Costa, H. H. (1987). Foraging behaviour and echolocation in the rufous horseshoe bat (Rhinolophus rouxi) of Sri Lanka. Behavioral Ecology and Sociobiology, 20(1), 53-67.

Norberg, U. M., \& Rayner, J. M. V. (1987). Ecological morphology and flight in bats (Mammalia; Chiroptera): wing adaptations, flight performance, foraging 
strategy and echolocation. Philosophical Transactions of the Royal Society of London B, 316(1179), 335-427.

Nosil, P., Crespi, B., \& Sandoval, C. (2003). Reproductive isolation driven by the combined effects of ecological adaptation and reinforcement. Proceedings of the Royal Society of London. Series B: Biological Sciences, 270(1527), 1911-1918.

Odendaal, L. J., \& Jacobs, D. S. (2011). Morphological correlates of echolocation frequency in the endemic cape horseshoe bat, Rhinolophus capensis (Chiroptera: Rhinolophidae). Journal of Comparative Physiology A, 197, 435-446.

Odendaal, L., Jacobs, D. S., \& Bishop, J. (2014). Sensory trait variation in an echolocating bat suggests roles for both selection and plasticity. BMC Evolutionary Biology, 14(1), 60.

Page, R. A., Ryan, M. J., \& Bernal, X. E. (2013). Be loved, be prey, be eaten. In K. Yasukawa (Ed.), Case studies: Integration and application of animal behavior: Vol. 3. Animal behavior (pp. 123-154). New York, NY: Praeger.

Pancratz, C. N., \& Cohen, L. B. (1970). Recovery of habituation in infants. Journal of Experimental Child Psychology, 9(2), 208-216.

Pennetier, C., Warren, B., Dabiré, K. R., Russell, I. J., \& Gibson, G. (2010). 'Singing on the wing' as a mechanism for species recognition in the malarial mosquito Anopheles gambiae. Current Biology, 20(2), 131-136.

Puechmaille, S. J., Borissov, I. M., Zsebok, S., Allegrini, B., Hizem, M., Kuenzel, S., et al. (2014). Female mate choice can drive the evolution of high frequency echolocation in bats: a case study with Rhinolophus mehelyi. PLoS One, 9(7), e103452.

Rankin, C. H., Abrams, T., Barry, R. J., Bhatnagar, S., Clayton, D. F., Colombo, J., et al. (2009). Habituation revisited: an updated and revised description of the behavioral characteristics of habituation. Neurobiology of Learning and Memory, 92(2), 135-138.

Ruczynski, I., Kalko, E. K., \& Siemers, B. M. (2007). The sensory basis of roost finding in a forest bat, Nyctalus noctula. The Journal of Experimental Biology, 210(20), 3607-3615.

Russ, J. M., Jones, G., Mackie, I. J., \& Racey, P. A. (2004). Interspecific responses to distress calls in bats (Chiroptera: Vespertilionidae): a function for convergence in call design? Animal Behaviour, 67(6), 1005-1014.

Russo, D., Mucedda, M., Bello, M., Biscardi, S., Pidinchedda, E., \& Jones, G. (2007). Divergent echolocation call frequencies in insular rhinolophids (Chiroptera): a case of character displacement? Journal of Biogeography, 34(12), 2129-2138.

Ryan, M. J., \& Rand, A. S. (1993). Species recognition and sexual selection as a unitary problem in animal communication. Evolution, 647-657.

Sandoval, L., Méndez, C., \& Mennill, D. J. (2013). Different vocal signals, but not prior experience, influence heterospecific from conspecific discrimination. Animal Behaviour, 85(5), 907-915.

Schnitzler, H.-U. (1968). Die Ultraschall-Ortungslaute der Hufeisen-Fledermäuse (Chiroptera-Rhinolophidae) in verschiedenen Orientierungssituationen. Zeitschrift für vergleichende Physiologie, 57(4), 376-408.

Schnitzler, H.-U., \& Kalko, E. K. V. (2001). Echolocation by insect-eating bats. BioScience, 51(7), 557-569.

Schnitzler, H.-U., Moss, C. F., \& Denzinger, A. (2003). From spatial orientation to food acquisition in echolocationg bats. Trends in Ecology and Evolution, 18(8), 386-394.

Schuchmann, M., \& Siemers, B. M. (2010). Behavioral Evidence for Community Wide Species Discrimination from Echolocation Calls in Bats. The American Naturalist, 176, 72-82.

Schuchmann, M., Puechmaille, S. B. J., \& Siemers, B. R. M. (2012). Horseshoe bats recognise the sex of conspecifics from their echolocation calls. Acta Chiropterologica, 14(1), 161-166.

Schuller, G., \& Pollak, G. (1979). Disproportionate frequency representation in the inferior colliculus of doppler-compensating greater horseshoe bats: evidence for an acoustic fovea. Journal of Comparative Physiology A, 132(1), 47-54.
Schuller, G., \& Suga, N. (1976). Storage of Doppler-shift information in the echolocation system of the CF-FM-bat, Rhinolophus ferrumequinum. Journal of Comparative Physiology A, 105(1), 9-14.

Seddon, N., \& Tobias, J. A. (2010). Character displacement from the receiver's perspective: species and mate recognition despite convergent signals in suboscine birds. Proceedings of the Royal Society B: Biological Sciences, 277(1693) 2475-2483.

Seyfarth, R. M., Cheney, D. L., \& Marler, P. (1980). Monkey responses to three different alarm calls: evidence of predator classification and semantic communication. Science, 210(4471), 801-803.

Siemers, B. M., Beedholm, K., Dietz, C., Dietz, I., \& Ivanova, T. (2005). Is species identity, sex, age or individual quality conveyed by echolocation call frequency in European horseshoe bats? Acta Chiropterologica, 7(2), 259-274.

Siemers, B. M., Kriner, E., Kaipf, I., Simon, M., \& Greif, S. (2012). Bats eavesdrop on the sound of copulating flies. Current Biology, 22(14), R563-R564.

Sikes, R. S., \& Gannon, W. L. (2011). Guidelines of the American Society of Mammalogists for the use of wild mammals in research. Journal of Mammalogy, 92(1), 235-253.

Simmons, A. M., Popper, A. N., Fay, R. R., \& Gerhardt, H. C. (2003). Acoustic communication (Vol. 16). New York, NY: Springer.

Slabbekoorn, H., \& Smith, T. B. (2002). Bird song, ecology and speciation. Philosophical Transactions of the Royal Society of London Series B, 357(1420), 493-503.

South African National Standard. (2008). The care and use of animals for scientific purposes, 10386 http://www.biologicalsciences.uct.ac.za/sites/default/files/ image_tool/images/75/files/SANS10386.pdf.

Thabah, A., Rossiter, S. J., Kingston, T., Zhang, S., Parsons, S., Mya Mya, K., et al. (2006). Genetic divergence and echolocation call frequency in cryptic species of Hipposideros larvatus s.l. (Chiroptera: Hipposideridae) from the Indo-Malayan region. Biological Journal of the Linnean Society, 88, 119-129.

Thomas, J. A., Moss, C. F., \& Vater, M. (2004). Echolocation in bats and dolphins. Chicago, IL: University of Chicago Press.

Tinbergen, N. (1952). 'Derived' activities; their causation, biological significance, origin, and emancipation during evolution. Quarterly Review of Biology, 27(1), $1-32$.

Tobias, J. A., \& Seddon, N. (2009). Sexual selection and ecological generalism are correlated in antbirds. Journal of Evolutionary Biology, 22(3), 623-636.

Tobias, J. A., Planqué, R., Cram, D. L., \& Seddon, N. (2014). Species interactions and the structure of complex communication networks. Proceedings of the National Academy of Sciences of the United States of America, 111(3), 1020-1025.

Vannoni, E., \& Mcelligott, A. G. (2007). Individual acoustic variation in fallow deer (Dama dama) common and harsh groans: a source-filter theory perspective. Ethology, 113(3), 223-234.

Vignal, C., \& Kelley, D. (2007). Significance of temporal and spectral acoustic cues for sexual recognition in Xenopus laevis. Proceedings of the Royal Society B: Biological Sciences, 274(1609), 479-488.

Vignal, C., Mathevon, N., \& Mottin, S. (2008). Mate recognition by female zebra finch: analysis of individuality in male call and first investigations on female decoding process. Behavioural Processes, 77(2), 191-198.

Voigt-Heucke, S. L., Taborsky, M., \& Dechmann, D. K. N. (2010). A dual function of echolocation: bats use echolocation calls to identify familiar and unfamiliar individuals. Animal Behaviour, 80(1), 59-67.

West-Eberhard, M. J. (1983). Sexual selection, social competition, and speciation. Quarterly Review of Biology, 58(2), 155-183.

Wilkins, M. R., Seddon, N., \& Safran, R. J. (2013). Evolutionary divergence in acoustic signals: causes and consequences. Trends in Ecology \& Evolution, 28(3), 156-166. 


\section{Appendix}

Table A1

Acoustic parameters (mean \pm SD and minima and maxima in parentheses, $N=$ no. of individuals, $n=$ no. of calls) measured on calls in the test playback files

\begin{tabular}{|c|c|c|c|c|c|c|}
\hline & RcaDH85 ( $N=9, n=147)$ & RcaHH84 ( $N=6, n=150)$ & RcaTF86 $(N=10, n=106)$ & RcaLS75 $(N=6, n=175)$ & $\operatorname{Rcl92}(N=4, n=223)$ & Rda86 $(N=6, n=105)$ \\
\hline Call duration (s) & $0.043 \pm 0.008(0.030-0.060)$ & $0.041 \pm 0.008(0.025-0.054)$ & $0.047 \pm 0.007(0.036-0.061)$ & $0.036 \pm 0.010(0.022-0.058)$ & $0.047 \pm 0.019(0.025-0.100)$ & $0.041 \pm 0.007(0.027-0.055)$ \\
\hline Interval (s) & $0.111 \pm 0.048(0.034-0.191)$ & $0.103 \pm 0.071(0.027-0.371)$ & $0.137 \pm 0.042(0.007-0.279)$ & $0.086 \pm 0.056(0.027-0.226)$ & $0.067 \pm 0.030(0.029-0.126)$ & $0.131 \pm 0.055(0.031-0.229)$ \\
\hline $\begin{array}{l}\text { Distance to maximum } \\
\text { frequency (s) }\end{array}$ & $0.025 \pm 0.005(0.016-0.036)$ & $0.025 \pm 0.005(0.014-0.033)$ & $0.028 \pm 0.006(0.014-0.039)$ & $0.021 \pm 0.006(0.009-0.039)$ & $0.030 \pm 0.018(0.014-0.082)$ & $0.022 \pm 0.006(0.012-0.033)$ \\
\hline Entropy & $0.116 \pm 0.008(0.107-0.137)$ & $0.111 \pm 0.004(0.107-0.120)$ & $0.117 \pm 0.003(0.112-0.124)$ & $0.124 \pm 0.008(0.112-0.14)$ & $0.148 \pm 0.009(0.134-0.165)$ & $0.123 \pm 0.008(0.113-0.146)$ \\
\hline $\begin{array}{l}\text { Frequency fundamental } \\
\text { harmonic }(\mathrm{kHz})\end{array}$ & $42.9 \pm 401(42.5-46.8)$ & $42.1 \pm 1050(41.2-46.1)$ & $42.6 \pm 259(42.2-43.2)$ & $37.3 \pm 726(34.0-38.7)$ & $45.8 \pm 329(42.4-46.1)$ & $42.4 \pm 439(41.6-43.4)$ \\
\hline $\begin{array}{l}\text { Peak frequency second } \\
\text { harmonic (= RF) }(\mathrm{kHz})\end{array}$ & $84.7 \pm 442(84.0-85.9)$ & $83.9 \pm 442(83.0-84.7)$ & $85.3 \pm 513(84.7-86.4)$ & $75.4 \pm 625(74.2-76.1)$ & $91.9 \pm 438(91.7-92.0)$ & $84.9 \pm 794(83.7-86.6)$ \\
\hline $\begin{array}{l}\text { Frequency third } \\
\text { harmonic }(\mathrm{kHz})\end{array}$ & $124.2 \pm 2664(112.5-125.0)$ & $112.9 \pm 2156(110.1-116.9)$ & 121. $5 \pm 2006(110.8-123.0)$ & $113.2 \pm 927(111.3-114.2)$ & $137.7 \pm 835(128.9-138.1)$ & $116.1 \pm 861(114.7-117.9)$ \\
\hline $\begin{array}{l}\text { Minimum frequency of } \\
\text { initial FM component } \\
\text { (second harmonic) } \\
(\mathrm{kHz})\end{array}$ & $79.1 \pm 2882(73.7-83.5)$ & $81.4 \pm 1339(78.3-83.0)$ & $80.7 \pm 3283(71.7-83.7)$ & $69.8 \pm 2220(65.6-74.4)$ & $79.4 \pm 4499(74.4-87.6)$ & $76.1 \pm 3297(71.5-82.7)$ \\
\hline $\begin{array}{l}\text { Minimum frequency of } \\
\text { final FM component } \\
\text { (second harmonic) } \\
(\mathrm{kHz})\end{array}$ & $74.1 \pm 4048(66.60-81.8)$ & $74.3 \pm 4092(68.1-80.5)$ & $71.5 \pm 1603(68.6-75.9)$ & $60.0 \pm 2945(47.8-64.4)$ & $65.3 \pm 2434(60.3-70.5)$ & $63.6 \pm 10687(29.7-73.7)$ \\
\hline Call rate (no./15 s) & 9.8 & 10.0 & 7.1 & 11.7 & 14.9 & 7.0 \\
\hline
\end{tabular}


Table A2

List of behaviours shown by the bats during the habituation - dishabituation experiments and coded in the video recordings

\begin{tabular}{|c|c|c|}
\hline Behaviour & Description & Category \\
\hline Calm & Bat shows no movement at all and hangs in a sleeping position on the perch & Inactive/Calm \\
\hline Relocate/settle down & Bat repositions its body usually accompanied by shaking slightly and ending in the sleeping position & Active \\
\hline Scrambling & Bat scrambles on the perch. Usually followed by settling down and ending in the sleeping position & Active \\
\hline Off perch & Bat leaves the perch, flying or scrambling & Active \\
\hline Fly towards loudspeaker & Bats flies off in a straight line towards the loudspeaker & Attentive \\
\hline Grooming & Bat grooms itself using its feet or licks its fur and wings & Active \\
\hline Wing stretching partly & Bat partly expands its wing(s) partly & Active \\
\hline Wing stretching complete & Bat completely expands its wing(s) & Active \\
\hline Single ear tip twitch & Bat slightly moves the tip of its ear(s) once & Active \\
\hline Series of single ear tip twitches & Bat slightly moves the tip of its ear(s) in a continuous series & Active \\
\hline Ears twitching slowly & Bat moves its entire ear(s) and the turns of the ear(s) are countable in real time & Attentive \\
\hline Ear twitching rapidly & Bat moves its entire ear(s). Movements are fast and single turns are not countable in real time & Attentive \\
\hline Scanning & Bat moves its entire ear(s) rapidly towards the sound source and has it head lifted up looking left and right & Attentive \\
\hline Head raised slightly & Bat lifts it head partly but the chin not visible & Attentive \\
\hline Head raised completely & Bat lifts its head completely looking up with its chin being visible & Attentive \\
\hline Contraction of leg completely & Bat contracts its leg(s) by bending its knees slightly & Attentive \\
\hline Contraction of leg slightly & Bat contracts it leg(s) completely by bending its knees fully & Attentive \\
\hline
\end{tabular}

